\title{
Poem
}

\section{The art of hovering}

No small feat, fifty wing beats per second, sixty, the tongue searching for sweetness, seventy. Territory matters,

beauty matters. In the right light, at the right angle, the throat shines. Recovery feels dependent on their brilliance, bones in mid-ear vibrate to their frequency. Learn the meaning, the magnitude of small and nothing and easy and little. The procedure is nothing, they said; the scars are small, there will be little pain. Try to swallow.

A hummingbird's heart is smaller than a pearl, larger than a grain of rice; it's nothing, just a little larger than nothing. Three hundred heartbeats per minute, check your pulse, five hundred, rub circles over scars, nine hundred, a thousand, try rising from the bed. Admire their acceleration, manoeuvres through acres of cherry and locust, predator and gust. Soon every move will cease to cause a flinch; you will adjust your vocabulary — no big deal — will refer to the surgery and smile.

But months later, long past summer, you hear echoes of the birds in midair and your heart beats faster

(although

like hovering

you appear

still)

Donna Steiner

Oswego, NY, USA.

donna.steiner@oswego.edu

doi: 10.5694/mjal4.01330 